\title{
Undulator radiation driven by laser-wakefield accelerator electron beams
}

\author{
S. M. Wiggins, M. P. Anania, G. H. Welsh, E. Brunetti, S. Cipiccia, P. A. Grant, D. Reboredo, \\ G. G. Manahan, D. W. Grant and D. A. Jaroszynski \\ Scottish Universities Physics Alliance, Department of Physics, University of Strathclyde, \\ Glasgow G4 0NG, United Kingdom
}

\begin{abstract}
The Advanced Laser-Plasma High-Energy Accelerators towards X-rays (ALPHA-X) programme is developing laser-plasma accelerators for the production of ultra-short electron bunches with subsequent generation of coherent, bright, shortwavelength radiation pulses. The new Scottish Centre for the Application of Plasma-based Accelerators (SCAPA) will develop a wide range of applications utilising such light sources. Electron bunches can be propagated through a magnetic undulator with the aim of generating fully coherent free-electron laser (FEL) radiation in the ultra-violet and X-rays spectral ranges. Demonstration experiments producing spontaneous undulator radiation have been conducted at visible and extreme ultra-violet wavelengths but it is an on-going challenge to generate and maintain electron bunches of sufficient quality in order to stimulate FEL behaviour. In the ALPHA-X beam line experiments, a Ti:sapphire femtosecond laser system with peak power $20 \mathrm{TW}$ has been used to generate electron bunches of energy 80-150 MeV in a $2 \mathrm{~mm}$ gas jet laser-plasma wakefield accelerator and these bunches have been transported through a 100 period planar undulator. High peak brilliance, narrow band spontaneous radiation pulses in the vacuum ultra-violet wavelength range have been generated. Analysis is provided with respect to the magnetic quadrupole beam transport system and subsequent effect on beam emittance and duration. Requirements for coherent spontaneous emission and FEL operation are presented.
\end{abstract}

Keywords: wakefield acceleration, laser-plasma interaction, relativistic electron beams, X-rays, radiation sources, undulator

\section{INTRODUCTION}

The laser wakefield accelerator (LWFA) is a very attractive alternative to radio frequency (RF) acceleration technology because of the roughly three orders of magnitude increase in the achievable accelerating gradient. ${ }^{1}$ Intense femtosecond duration laser pulses are focused into gas or plasma targets resulting in the formation of highly relativistic electron bunches with enticing properties, given that the accelerator is so short (mm-cm scale): energy of 100 s of $\mathrm{MeV}$ up to $4 \mathrm{GeV}$, ${ }^{2}$ energy spread of less than $1 \%,{ }^{3}$ transverse normalised emittance less than $0.5 \pi \mathrm{mm}$ mrad, $^{4}$ few femtosecond duration ${ }^{5}$ and kiloAmpere peak currents. ${ }^{3,5}$

LWFA electron beams can serve as sources of very bright radiation. This can be internal to the accelerator itself, that is, $\mathrm{X}$-ray and gamma-ray betatron radiation ${ }^{6}$ or external and downstream of the accelerator if the electron beam is transported to create gamma-ray bremsstrahlung radiation in a metal target ${ }^{7}$ or undulator radiation in a magnetic array. ${ }^{8}$ Such radiation sources are tunable, compact and brilliant and offer huge potential for the relevant user communities.

One clear route for the generation of bright, coherent radiation is to achieve free-electron laser (FEL) action akin to today's X-ray FELs such as LCLS ${ }^{9}$ and SACLA. ${ }^{10}$ These machines are based on conventional RF technology and, therefore, are km-scale infrastructures. Miniaturisation of the accelerator using laser-plasmas would greatly reduce the infrastructure requirements and greatly increase accessibility to such light sources. One of the goals of the ALPHA-X programme is to develop LWFA-driven FELs at short wavelength which, in the near future, will capitalise on the newly built Scottish Centre for the Application of Plasma-based Accelerators (SCAPA) at the University of Strathclyde as a test bed for the development. 
In this paper, we present experimental progress into the generation of radiation in a magnetic undulator-based with a view towards FEL operation. The two key concepts are electron beam quality, that must fulfil certain criteria, and beam transport to maintain beam quality from source to undulator. On the ALPHA-X Beam Line, electron beams have been fully characterised in terms of their energy spectrum, charge, transverse emittance and bunch length. Comparisons are made between simulations and measurements of the beam transport system. Experiments are conducted with relatively low electron beam energy that produces ultra-violet radiation in the undulator where FEL criteria will be more readily fulfilled than at X-ray wavelengths.

\section{ALPHA-X BEAM LINE EXPERIMENTAL SETUP}

Experiments to produce high quality electron beams with a view towards FEL operation are being conducted on the ALPHA-X laser-wakefield accelerator beam line. ${ }^{11}$ The experimental setup is shown in Figure 1. Electrons are accelerated in a relativistically self-guiding plasma channel formed in a helium gas jet by a Ti:sapphire laser pulse (wavelength $\lambda_{0}=800$ $\mathrm{nm}$, duration $=36 \mathrm{fs}$ ). The gas jet nozzle is of length $2 \mathrm{~mm}$ and the plasma density $n_{e} \approx 1 \times 10^{19} \mathrm{~cm}^{-3}$. The laser pulse is focused to a spot size of $40 \mu \mathrm{m}\left(1 / e^{2}\right.$ diameter) by an F/16 spherical mirror. Therefore, at the focus, the peak intensity $I=2 \times$ $10^{18} \mathrm{~W} \mathrm{~cm}^{-2}$ and the normalised vector potential $a_{0}=e E /\left(m_{0} c \omega_{0}\right)=1.0$ where $e$ and $m_{0}$ are the electron charge and rest mass respectively, $E=\left(2 I / c \varepsilon_{0}\right)^{1 / 2}$ is the peak electric field of the laser pulse, $c$ is the speed of light, $\varepsilon_{0}$ is the permittivity of free space and $\omega_{0}=2 \pi c / \lambda_{0}$ is the laser frequency.

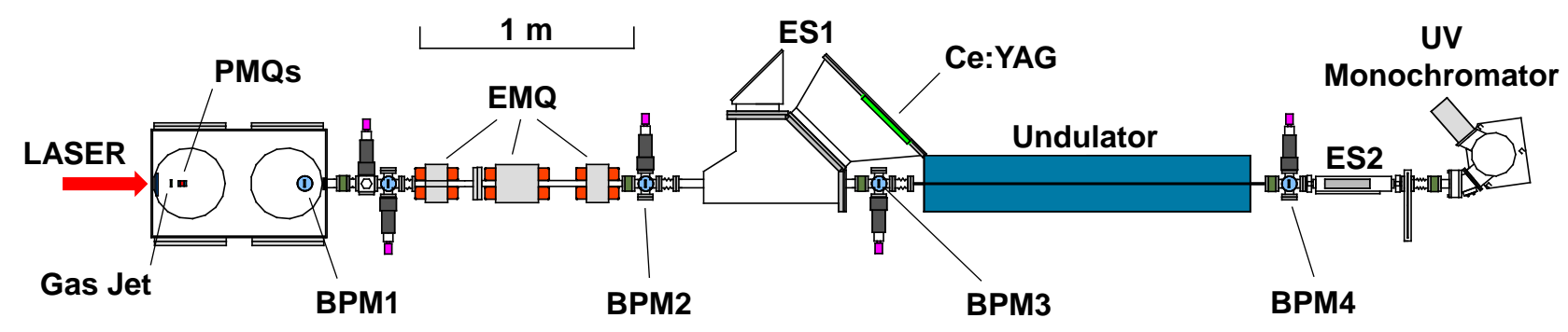

FIGURE 1. The ALPHA-X wakefield accelerator beam line. PMQs denote permanent magnet quadrupoles, EMQ denotes electromagnet quadrupoles and BPM denote Lanex beam profile monitors. ES1 and ES2 are the electron spectrometers.

Electrons beams emitted from the plasma are imaged at various locations along the beam line using a series of pop-in beam profile monitors (BPMs comprising Lanex screens). Beam transport pertains to a triplet of miniature permanent magnetic quadrupoles (PMQs) ${ }^{12}$ located directly after the gas jet and to a triplet of electromagnet quadrupoles (EMQs) further downstream, see Figure 1. The electron energy spectrum is primarily measured using a magnetic dipole imaging spectrometer with maximum field strength of $1.8 \mathrm{~T}$ that enables good energy resolution over a wide energy range. Ce:YAG crystal is the scintillation material in this electron spectrometer.

The undulator is located after the main electron spectrometer. It features a slotted pole planar design ${ }^{13}$ with a pole gap distance of $8 \mathrm{~mm}$. The peak on-axis magnetic field strength $\mathrm{B}_{\mathrm{u}}=0.27 \mathrm{~T}$ and undulator deflection parameter $\mathrm{K}=0.38$. Overall length is $1.5 \mathrm{~m}$ with $\mathrm{N}_{\mathrm{u}}=100$ periods and period $\lambda_{\mathrm{u}}=15 \mathrm{~mm}$. Distance from accelerator exit to undulator entrance is $3.52 \mathrm{~m}$. After the undulator is a crude yet effective compact bending magnet dipole spectrometer. This acts to dump the electron beam ahead of the radiation diagnostics as well as provide a measurement (Lanex screen scintillation) of the electron spectrum after propagation through the undulator. Absolute charge is obtained using imaging plates and cross-calibration techniques are used with respect to the beam position monitors and electron spectrometer scintillation screens.

Undulator output radiation is detected using a vacuum scanning monochromator (with platinum-coated toroidal mirror and 300 lines/mm grating) and 16-bit CCD camera. The grating is centred on $220 \mathrm{~nm}$ with resolution of about $5 \mathrm{~nm}$. The toroidal mirror has a peak reflectivity of $65 \%$ and the quantum efficiency of the camera is $25 \%$ across the relevant spectral 
range. Plasma emission and laser light has been blocked by an aluminium foil (thickness $800 \mathrm{~nm}$ ) positioned at the beam position monitor location just before the undulator, as described later.

\section{ALPHA-X BEAM TRANSPORT}

The PMQ settings are optimised for an electron energy of $130 \mathrm{MeV}$. Distance from gas jet exit to PMQ entrance is $30 \mathrm{~mm}$ and the PMQ triplet pitch is $55 \mathrm{~mm}$. The aim of this setting is to produce a collimated beam out of the permanent quadrupoles when the electron energy is $130 \mathrm{MeV}$, which will then be focused at the centre of the undulator with the EMQs. The expected beam sizes, produced using the GPT code, ${ }^{14}$ can be compared to the measured beam sizes where example beam profile monitor images are shown in Figure 2. Complications arise in the analysis because of the shot-to-shot variations in the electron beam energy, over a fairly broad range of 90-160 MeV, as shown in Figure 3. Hence, simulations have been conducted for a corresponding energy range as shown in Figure 4.
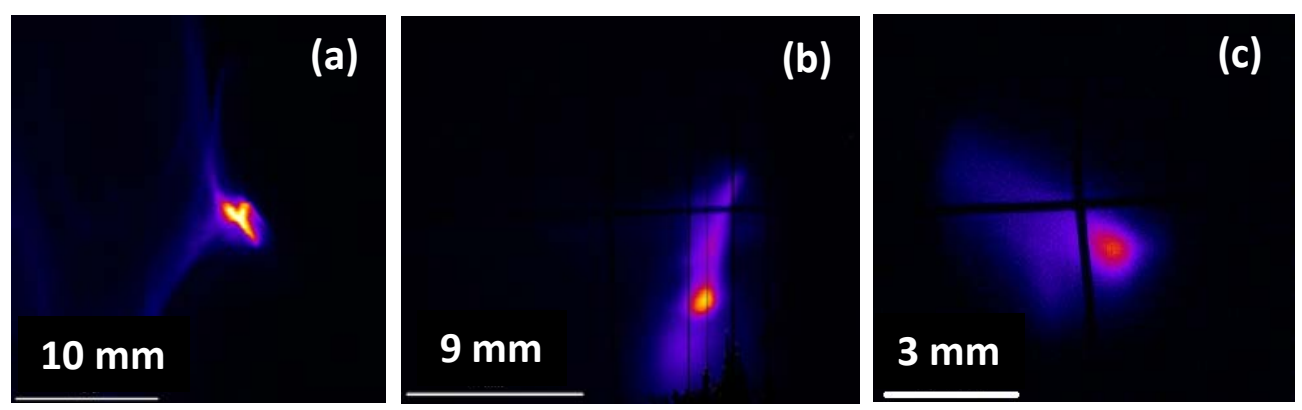

FIGURE 2. Example images at BPM1, BPM3 and BPM4.
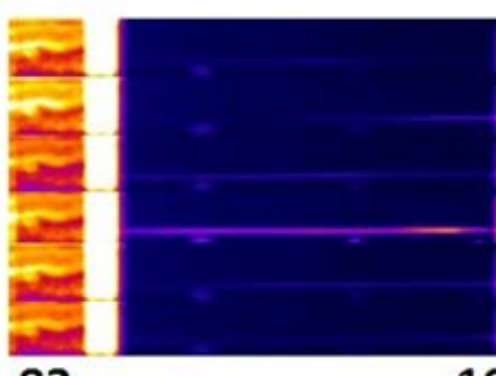

83

108

149

222

Energy (MeV)

FIGURE 3. Example six consecutive electron spectra captured on electron spectrometer ES1. The bright signal at the lower energy extreme is due to noise.

Simulations predict good transport of the electron beam through the undulator with beam radius in each transverse plane in the range $\sim 200-300 \mu \mathrm{m}$ in the region of the design energy. This implies a focal spot waist down to $\sim 150 \mu \mathrm{m}$ close to the undulator centre ${ }^{12}$ and beta function approximately equal to the undulator length. The experimental results indicated in Figure 4 each represent 50 consecutive shots with average beam energy of $(120 \pm 40) \mathrm{MeV}$. The respective mean beam radii are larger than the simulated radii in the region of the design energy, however, the smallest measured radii either side of the undulator (BPM3 and BPM4) are $\sim 300 \mu \mathrm{m}$ in each transverse plane which is in reasonably good agreement with the simulations. This suggests that beam transport is reasonably good when the electron beam energy coincides with the PMQ energy setting. 

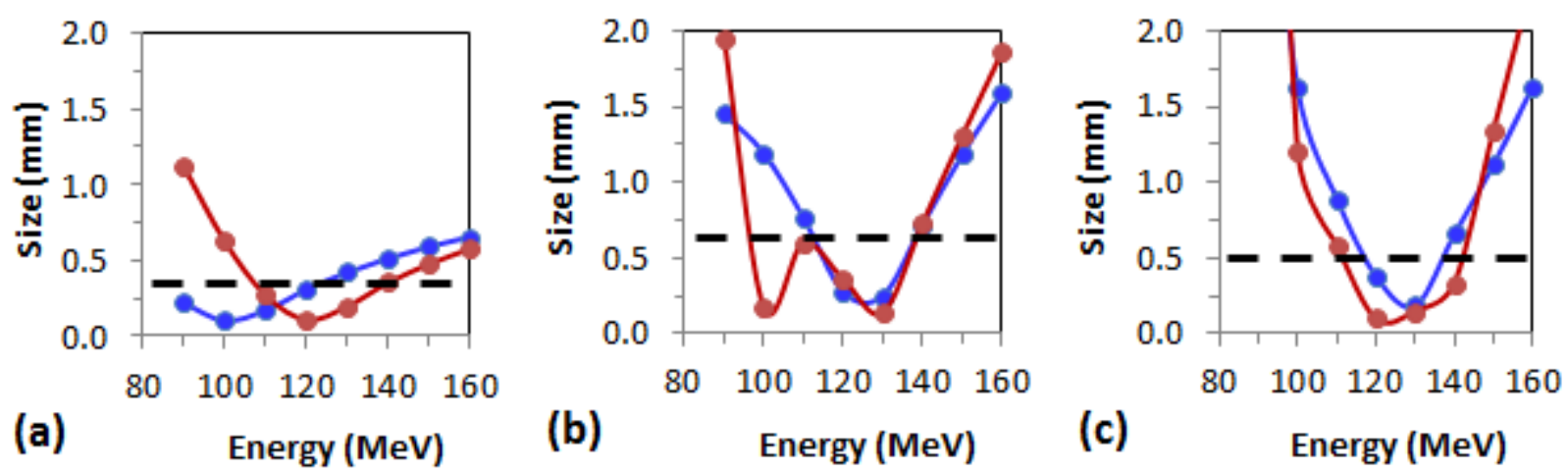

FIGURE 4. Simulated rms beam radii (horizontal: blue, vertical: red) for BPM locations (a) BPM1, (b) BPM3 and (c) BPM4 where the horizontal dashed lines indicate the corresponding experimental measurements of the mean beam size (averaged over both directions).

Other measurements have reported on the electron beam quality. The transverse normalised emittance has been characterised by pepper-pot masks and detection system resolution sets an upper limit of $\sim 1.0 \pi \mathrm{mm}$ mrad. ${ }^{15}$ Infra-red transition radiation measurements have enabled rms bunch length estimates down to 1.5 fs to be made. ${ }^{16}$ The beam quality upon transportation though can be degraded by the filter used to block the laser and plasma emission. Shown in Figure 5 are typical electron beam profiles at BPM3 for two different locations for a thick beam block (50 $\mu \mathrm{m}$ thick aluminium): more upstream at $700 \mathrm{~mm}$ after the accelerator and more downstream at $3068 \mathrm{~mm}$ after the accelerator. The resulting mean beam radii were $1.78 \mathrm{~mm}$ and $0.61 \mathrm{~mm}$ respectively, i.e., a near factor of 3 increase in the beam size at BPM3 caused by the beam block being located farther upstream. Further Monte Carlo simulations have detailed the increase in emittance due to this scattering element in the beam line. It is found that the degree of emittance growth increases as the beam block location recedes from the beam focal position. The latter beam block position has been determined as the most practical given its proximity to the undulator and the thickness of the aluminium block has been minimised to $800 \mathrm{~nm}$.
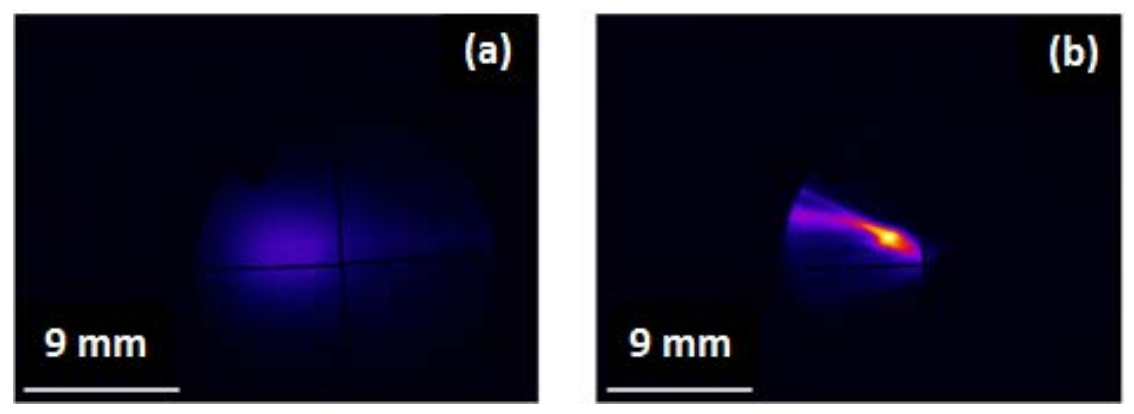

FIGURE 5. Example BPM3 images at (a) upstream and (b) downstream beam block locations.

\section{UNDULATOR RADIATION MEASUREMENTS}

Figure 6 displays example undulator radiation spectra with the corresponding spectra of the electron beam that has propagated through the undulator to be imaged in the beam dump spectrometer ES2. The absolute energy of electrons as measured here is in good agreement with the main electron spectrometer ES1 while the transportation of the "main peak" of electrons (discounting lower energy shoulders or pedestals) is reasonable with $\sim 30 \%$ beam loss from BPM3 to ES2. The mean charge on ES2 is $(0.8 \pm 0.4) \mathrm{pC}$. 

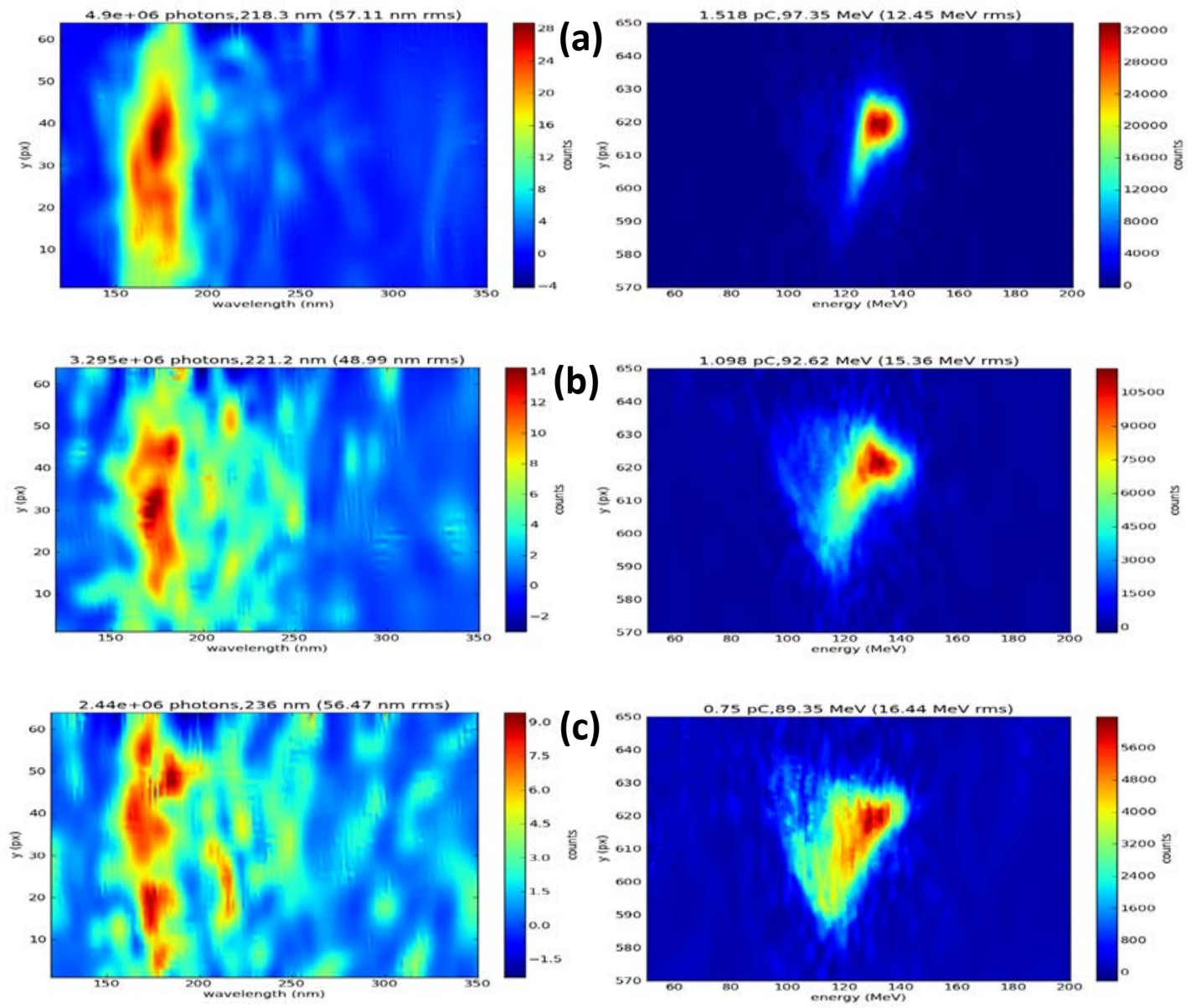

FIGURE 6. Example undulator radiation and electron spectra (on ES2) captured simultaneously giving (a) $4.9 \times 10^{6}$ measured photons at $1.5 \mathrm{pC}$ charge, (b) $3.3 \times 10^{6}$ measured photons at $1.1 \mathrm{pC}$ charge and (c) $2.4 \times 10^{6}$ measured photons at $0.8 \mathrm{pC}$ charge.

In agreement with undulator theory, ${ }^{17}$ scaling of the peak radiation wavelength as the inverse of the square of the electron energy is obtained [Figure 7(a)], although, for energy greater than $\sim 110 \mathrm{MeV}$, the radiation peak is skewed by detection system drop-off below $180 \mathrm{~nm}$ and there may also be detection of the third order of the second harmonic. The fundamental radiation wavelength would be expected to extend down to $\sim 15 \mathrm{~nm}$ at the highest electron energy (135 MeV) in the data set. The mean electron energy (102 \pm 8) MeV was actually lower than the PMQ design energy of $130 \mathrm{MeV}$, therefore, the mean electron bunch duration at the undulator centre is estimated to be $28 \mathrm{fs}$, as opposed to $3 \mathrm{fs}$ at the design energy. The mean radiation spectral bandwidth of (32 \pm 7 ) \%, that is related to the electron beam properites, ${ }^{18}$ is seen to be dominated by the electron energy spread of $\sim 15 \%$ measured on ES2. It appears that a large fraction of the radiation has been generated by pedestal electrons lost through the undulator (compare electron spectra of Figure 3 and Figure 6).

The fact that the radiation is incoherent synchrotron-like spontaneous emission is confirmed by the linear scaling obtained for the number of detected photons as a function of the beam charge [Figure 7(b)]. Enhanced coherence arising from coherent spontaneous emission ${ }^{19}$ or FEL action would be evidenced by a non-linear scaling. Clearly, beam quality in the undulator has not been optimised, in terms of the emittances or bunch length (peak current is up to 350 A only). Nevertheless, the total number of detected photons is up to $9 \times 10^{6}$ resulting in a mean peak brilliance of $1 \times 10^{18}$ photons $/ \mathrm{s} / \mathrm{mrad}^{2} / \mathrm{mm}^{2} / 0.1 \%$ bandwidth (based on the measured radiation divergence of $2 \mathrm{mrad}$, estimated source diameter of 
$300 \mu \mathrm{m}$, and mean rms duration of $100 \mathrm{fs}$ ). The relatively long radiation pulse duration is a sum of the mean electron duration (28 fs simulated) and mean radiation slippage duration (72 fs).
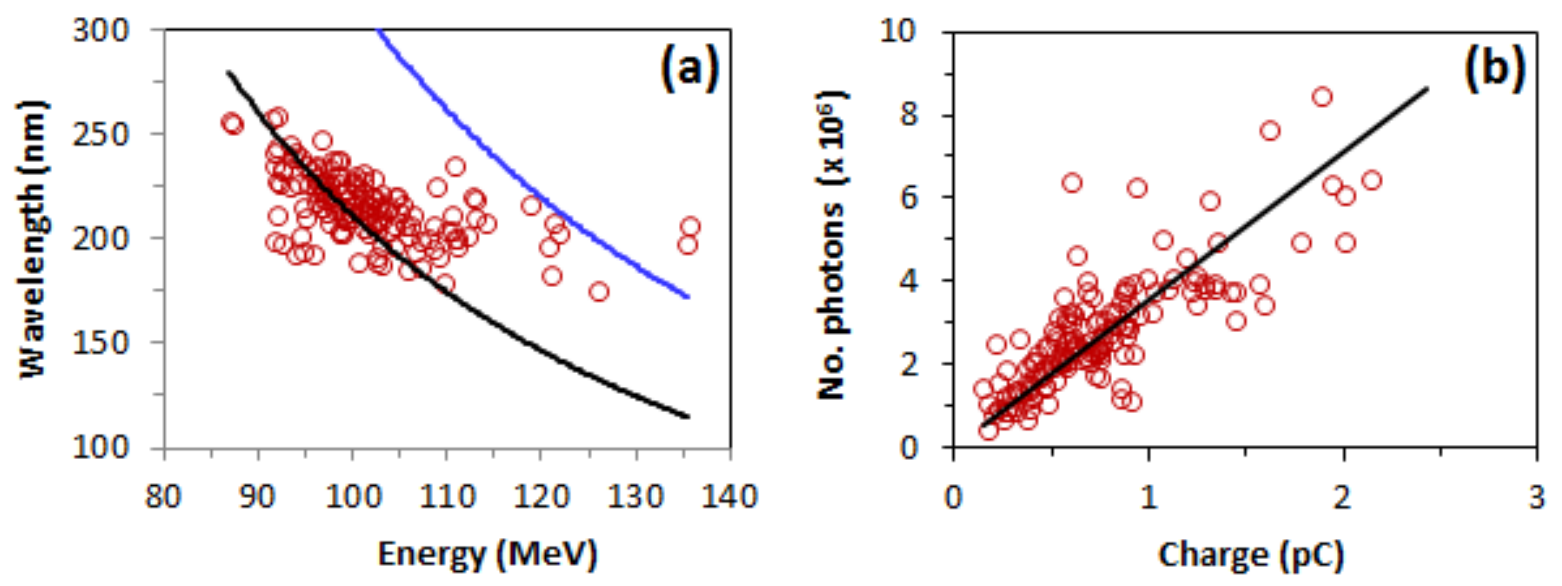

FIGURE 7. Undulator radiation (a) peak wavelength as a function of electron energy, where the black and blue curves are the theoretical dependences for the fundamental and second harmonic ( $3^{\text {rd }}$ order) respectively, and (b) number of detected photons as a function of beam charge where the black line is a linear best-fit.

\section{CONCLUSIONS}

A bright tunable source of ultrashort pulse ultra-violet radiation, driven by a LWFA electron beam, has been demonstrated. Emission is limited to spontaneous undulator radiation because of the relatively long electron bunch length (lowering the peak current) and large energy spread. A rule-of-thumb for FEL operation in this spectral range is to obtain $\sim 1 \mathrm{kA}$ peak current and 1\% energy spread. Transverse emittance does not have such a restrictive criterion and the use of a thin aluminium beam block as close as possible to the beam focal position is seen to not greatly degrade the transverse emittance. Quadrupole settings need to be optimised for the given electron energy to maintain peak current while energy spread can be minimised by employing, for example, self-injection close to threshold ${ }^{16}$ for the electron beam production.

Coherent spontaneous emission could also be driven by Fourier components at the radiation wavelength and this could eventually seed FEL operation. Longer wavelength systems have demonstrated that bunch rise times $\sim 10 \lambda / c$, where $\lambda$ is the radiation wavelength, are sufficiently rapid to seed self-amplified coherent spontaneous emission (SACSE) in FELs. ${ }^{20,21}$ With optimal beam transport that maintains a bunch duration of less than $\sim 5$ fs to the undulator, then the leading edge of the bunch or significant sub-structure ${ }^{22}$ could act as coherent seed on the scale of the radiation wavelength.

\section{ACKNOWLEDGEMENTS}

We acknowledge support of the U.K. EPSRC (grant no. EP/J018171/1), the EC's LASERLAB-EUROPE (grant agreement no. 284464, Seventh Framework Programme), EuCARD-2 (grant no. 312453, FP7) and the Extreme Light Infrastructure (ELI) European Project. We thank D. Clark and T. McCanny for technical support.

\section{REFERENCES}

[1] Esarey, E., Schroeder, C. B. and Leemans, W. P., "Physics of laser-driven plasma-based electron accelerators," Rev. Mod. Phys. 81, 1229 (2009).

[2] Leemans, W. P. et al., "Multi-GeV Electron Beams from Capillary-Discharge-Guided Subpetawatt Laser Pulses in the Self-Trapping Regime,” Phys. Rev. Lett. 113, 245002 (2014).

[3] Wiggins, S. M. et al., "High quality electron beams from a laser wakefield accelerator," Plasma Phys. Control. Fusion 52, 124032 (2010). 
[4] Weingartner, R. et al., "Ultralow emittance electron beams from a laser-wakefield accelerator,” Phys. Rev. ST Accel. Beams 15, 111302 (2012).

[5] Lundh, O. et al., "Few femtosecond, few kiloampere electron bunch produced by a laser-plasma accelerator,” Nature Phys. 7, 219 (2011).

[6] Cipiccia, S. et al., “Gamma-rays from harmonically resonant betatron oscillations in a plasma wake,” Nature Phys. 7, 867 (2011).

[7] Cipiccia, S. et al., “A tuneable ultra-compact high-power, ultra-short pulsed, bright gamma-ray source based on bremsstrahlung radiation from laser-plasma accelerated electrons,” J. Appl. Phys. 111, 063302 (2012).

[8] Schlenvoigt, H.-P. et al., "A compact synchrotron radiation source driven by a laser-plasma wakefield accelerator," Nature Phys. 4, 130 (2008).

[9] Emma, P. et al., “First lasing and operation of an ångstrom-wavelength free-electron laser,” Nature Photon. 4, 6417 (2010).

[10] Ishikawa, T. et al., “A compact X-ray free-electron laser emitting in the sub-ångström region,” Nature Photon. 6, 540 (2012).

[11] Jaroszynski, D. A. et al., "Radiation sources based on laser-plasma interactions,” Phil. Trans. R. Soc. A 364, 689 (2006).

[12] Anania, M. P. et al., “Transport of ultra-short electron bunches in a free-electron laser driven by a laser-plasma wakefield accelerator,” Proc. SPIE 7359, 735916 (2009).

[13] Shepherd, B. J. A. and Clarke, J. A., "Design, measurement and correction of a pair of novel focusing undulators for the ALPHA-X project,” Nucl. Instrum. Methods Phys. Res. Sect. A 654, 8 (2011).

[14] van der Geer, S. B., Luiten, O. J., de Loos, M. J., Poeplau, G. and van Rienen, U., “3D space-charge model for GPT simulations of high brightness electron bunches," in [Institute of Physics Conference Series No. 175], Institute of Physics, Bristol, UK, 101 (2005).

[15] Manahan, G. G. et al., "Characterization of laser-driven single and double electron bunches with a permanent magnet quadrupole triplet and pepper-pot mask,” New J. Phys. 16, 103006 (2014).

[16] Islam, M. R. et al., "Near-threshold electron injection in the laser-plasma wakefield accelerator leading to femtosecond bunches," submitted.

[17] Rebernik Ribic, P. and Margaritondo, G., "Status and prospects of x-ray free-electron lasers (X-FELs): a simple presentation,” J. Phys. D: Appl. Phys. 45, 213001 (2012).

[18] Gallacher, J. G. et al., “A method of determining narrow energy spread electron beams from a laser plasma wakefield accelerator using undulator radiation,” Phys. Plasmas 16, 093102 (2009).

[19] McNeil, B. W. J. and Jaroszynski, D. A., "Self-amplification of coherent spontaneous emission in the free electron laser,” Opt. Commun. 165, 65 (1999).

[20] Jaroszynski, D. A., Bakker, R. J., van der Meer, A. F. G., Oepts, D. and Amersfoort, P. W. “Coherent startup of an infrared free-electron laser,” Phys. Rev. Lett. 71, 3798 (1993).

[21] Wiggins, S. M. et al., "Self-Amplification of Coherent Spontaneous Emission in a Cherenkov Free-Electron Maser,” Phys. Rev. Lett. 84, 2393 (2000).

[22] Lin, C. et al., "Long-Range Persistence of Femtosecond Modulations on Laser-Plasma-Accelerated Electron Beams," Phys. Rev. Lett. 108, 094801 (2012). 